\title{
Study and Development of Home Interaction Terminal
}

\author{
Yeshen $\mathrm{He}^{1, \mathrm{a}}$, Chun Wang ${ }^{1, \mathrm{~b}}$, Daoyuan Wang ${ }^{1, \mathrm{c}}$, Lin $\mathrm{Mu}^{1, \mathrm{~d}}$, Jiahua $\mathrm{Wu}^{2, \mathrm{e}}$ \\ 1.NARI Group, China Gridcom CO,.LTD \\ Shenzhen, China \\ ${ }^{2}$ Class 5 ,Grade 2011, Institute of electrical and information engineering of Hunan university \\ Changsha, China \\ aheyeshen@sgepri.sgcc.com.cn, bwangchun@sgepri.sgcc.com.cn, cwangdaoyuan@sgepri.sgcc.c \\ om.cn, \\ dmulin@sgepri.sgcc.com.cn, ${ }^{\mathrm{e}}$ nelsonhonger@189.cn
}

Keywords:power line carrier; electric energy data acquisition; smart interaction system; home interaction terminal

\begin{abstract}
In order to improve the level of interaction service between users and grids, and dig into the capacity of smart meters, this paper developed the home interaction terminal. It meets people's interactive demands, which plays an important role in the smart interaction system. Based on the electric energy data acquisition system, the smart interaction system is composed of home interaction terminal, electronic equipment of users, smart socket and smart home. In this paper, the key technology and major problems of home interaction terminal were researched and analyzed. And an experimental demonstration system of smart interaction system was set up. Test result showed that electric energy data acquisition system could be transformed to smart interaction system, and verified the superiority of home interaction terminal.
\end{abstract}

\section{Introduction}

Electric energy data acquisition system is the important part of the construction of smart grid, which has the advantage of low cost, wide coverage, and ease use for customs[1,2]. Because of the advantage of wide bandwidth and high communication rate, power line broadband carrier communication can achieve real time concurrent transmission, and it could be used for electric energy data acquisition system. And power line broadband carrier communication predominates at aspects of rate, bandwidth, the capability of noise immunity and anti-attenuation, and impedance[3].

The main features of smart grid interaction[4,5] are that users can get the information of electricity and electric charge in time. And users hope that they can get a recommended solution on family's electricity. So, in this paper, we had study and development on the Home Interaction Terminal. As well as, home interaction terminal, smart socket and home, and electric energy data acquisition system formed the smart interaction system, which had the characteristic of simple, wide coverage, superior communication performance.

In this paper, firstly, we analyzed the superiority of home interaction terminal based on the electric energy data acquisition system by power line broadband carrier communication, and gave the structure of smart interaction system. Secondly, we highlighted the function and the design of software and hardware of home interaction terminal. Lastly, we set up an experimental demonstration system of smart interaction system, whose result showed that electric energy data acquisition system could be transformed to smart interaction system, and verified the superiority of home interaction terminal.

\section{Study on the smart interaction system}

The smart interaction system includes home interaction terminal, electronic equipment of users, smart socket, and smart meter. There are two communication ways(power line broadband carrier and Micro-Power Wireless) in the electric energy data acquisition system[6]. And home interaction 
terminal can communicate with smart meter, concentrator and the master station. According to the different communication ways and coverage, this article discussed four solutions in the table 1 . From the table 1 , we knows that plans $\mathrm{C}$ and $\mathrm{D}$ have advantages in information type and economics, but they are at a disadvantage compared to plans A and B in system complexity, the effect to electric energy data acquisition system, and the modification of communication protocol. And with the increase of interaction data, the system properties will decrease. Compared to plan B, plan A predominates at aspects of communication properties, coverage, and support capability.

TABLE I. THE COMPARISON OF FOUR PLANS

\begin{tabular}{|c|c|c|c|c|c|}
\hline NO & Items & Plan A & Plan B & Plan C & Plan D \\
\hline 1 & Key points & $\begin{array}{l}\text { 1)communication } \\
\text { mode is Broadband } \\
\text { Power Line } \\
\text { 2)communication } \\
\text { between terminal } \\
\text { and smart meter }\end{array}$ & $\begin{array}{l}\text { 1)communication } \\
\text { mode is Micro- } \\
\text { Power Wireless } \\
\text { 2)communication } \\
\text { between terminal } \\
\text { and smart meter }\end{array}$ & $\begin{array}{l}\text { 1)communication } \\
\text { mode is Broadband } \\
\text { Power Line } \\
\text { 2)communication } \\
\text { between terminal } \\
\text { and concentrator or } \\
\text { master station }\end{array}$ & $\begin{array}{l}\text { 1)communication } \\
\text { mode is Micro- } \\
\text { Power Wireless } \\
\text { 2)communication } \\
\text { between terminal } \\
\text { and concentrator or } \\
\text { master station }\end{array}$ \\
\hline 2 & $\begin{array}{c}\text { Acquisition meters } \\
\text { information }\end{array}$ & $(1)$ & $\theta$ & 0 & 0 \\
\hline 3 & $\begin{array}{c}\text { Acquisition other } \\
\text { information }\end{array}$ & X & X & 0 & 0 \\
\hline 4 & $\begin{array}{l}\text { Influence on the } \\
\text { electric energy data } \\
\text { acquisition system }\end{array}$ & Small & Small & Great & Great \\
\hline 5 & $\begin{array}{c}\text { Protocol } \\
\text { modification }\end{array}$ & No & No & Yes & Yes \\
\hline 6 & Data privacy & High & Low & High & Low \\
\hline 7 & $\begin{array}{l}\text { Bandwidth and } \\
\text { delay }\end{array}$ & Excellent & Good & Excellent & Good \\
\hline 8 & Coverage & Large & Medium & Large & Medium \\
\hline 9 & Complexity & Low & Medium & High & High \\
\hline 10 & Transformation costs & Smart meter & Smart meter & $\begin{array}{l}\text { Concentrator and } \\
\text { master station }\end{array}$ & $\begin{array}{l}\text { Concentrator and } \\
\text { master station }\end{array}$ \\
\hline
\end{tabular}

In the smart interaction system, a broadband power line carrier communication module is installed on the smart meter, and the module can send the power-expenditure information to the power line. At the same time, home interaction terminal converts power line signals into WIFI signals, and interacts with the users by electronic devices. On the other side, users can control the smart socket and home by home interaction terminal. The structure of smart interaction system is shown in Figure 1.

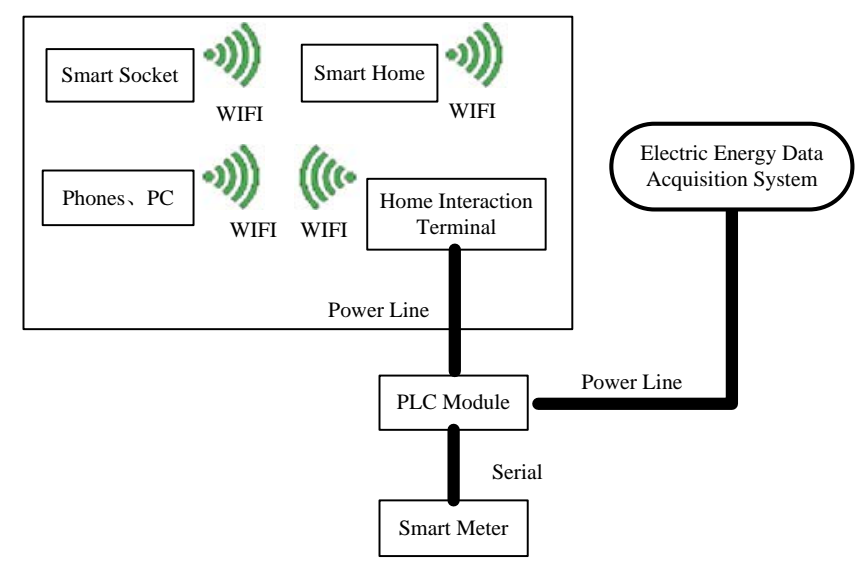

Figure 1. The structure of smart interaction system.

\section{The exploitation of home interaction terminal}

\section{A. Hardware}

As the key products, on the one hand, home interaction terminal can provide service and information about smart meters for users. On the other hand, it can control the electric equipments according to the users' orders. The hardware of home interaction terminal mainly includes AC/DC 
power, LCM (local communication module, LCM), MCU, WIFI module, ESAM module, lights, buttons and so on. Figure 2 shows the basic structure of home interaction terminal. WIFI module can achieve the connection between home interaction terminal and smart home, smart phone, and home interaction terminal. And users can get the power-expenditure information and control the electric equipments through WIFI network. ESAM module ensure that local data can be transmitted securely. AC/DC module can convert AC $220 \mathrm{~V}$ power into DC $12 \mathrm{~V}$ power, and supply power for the system. On the one hand, AC power port supplies source for the system by switch power circuit. On the other hand, it is a connection between power line coupler circuit and LCM.

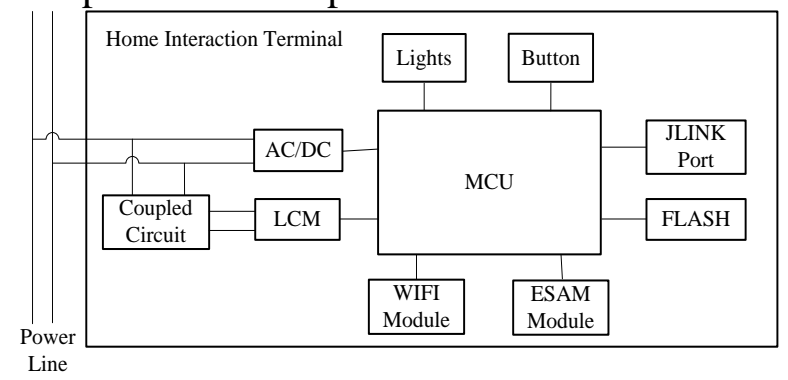

Figure 2. The basic structure of home interaction terminal.

LCM can obtain smart meters' power-expenditure information, and achieve interaction between power line broadband carrier module and home interaction terminal. LCM consists of SG5000, FLASH, and kinds of interface circuits. Firstly, the MCU data is converted into OFDM signal through data framing, encryption, encoding and so on by LCM. Secondly, the OFDM signal can transmit on the power line trough the coupled circuit. Meanwhile, LCM can receive the OFDM signal from power line through the coupled circuit. And it converts the OFDM signal into the MCU data by digital-to-analogue conversion, demodulation, decoding and so on. Then the MCU data can be sent to MCU by UART.

\section{B. Software}

In the smart interaction system, the data flow interacts through the serial between smart meter and PLC(power line carrier, PLC) module. The data flow interacts through the power line between PLC module and home interaction terminal. And home interaction terminal converts the data flow from PLC module into WIFI data flow. The data flow of smart interaction system is shown in the figure 3. In the home interaction terminal, the data flow encryption interaction between MCU and ESAM avoids interference from the electric energy data acquisition system. The data flow of home interaction terminal is shown in the figure 4.

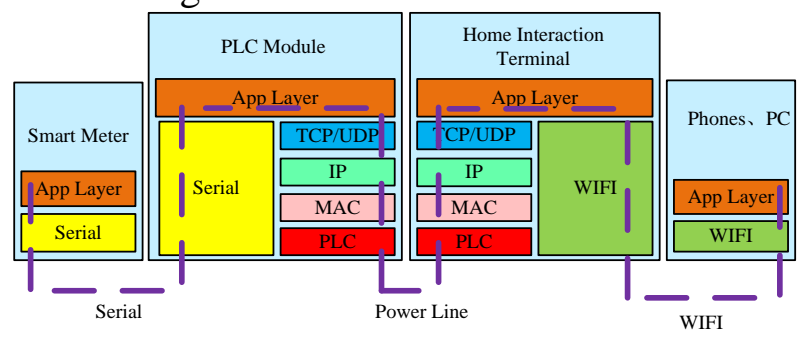

Figure 3. The data flow of smart interaction system.

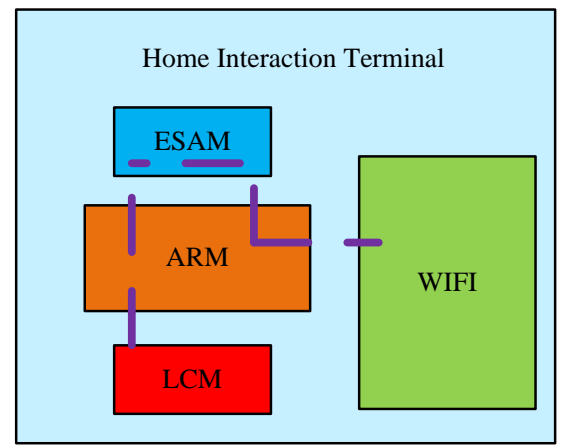

Figure 4. The data flow of home interaction termainal. 
There is a crucial factor when the home interaction terminal for information interaction in the first time. Home interaction terminal and PLC module must be certified by ESAM. It is not necessary for ESAM certification when other information have interaction between home interaction terminal and PLC module. In the process of interaction, ESAM will encrypt the information before sending. Figure 5 shows the specific information interaction process.

1)Users input ESAM certification orders, and submit them to home interaction terminal.

2)Home interaction terminal loads the current operation steps. If the steps are loaded successfully, ESAM certification will be executed. If the steps fails to load, the program will return failure information.

3)If the ESAM certification passes, information interaction will be completed successfully. Then the system submits operation results to users and processes other information interaction. If the authentication fails to pass, the program will generate interaction window and remind users that ESAM certification fails to pass.

4)Users can input the orders of electric energy data acquisition, control command of samrt socket after ESAM certification passes. In the process of interaction, ESAM will encrypt the information which users input.

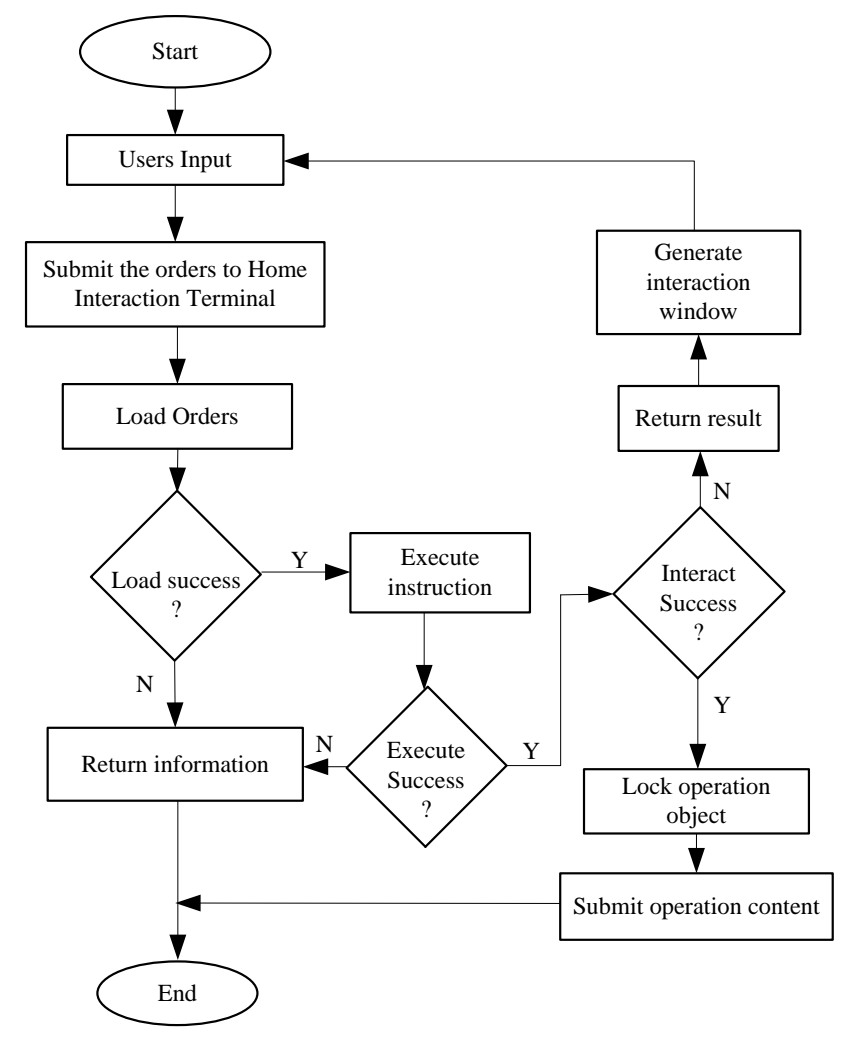

Figure 5. The specific information interaction process.Comparative test of performance of home interaction terminal

\section{Comparative test of performance of home interaction terminal}

In this paper, the smart interaction system consisted of home interaction terminal and users' electric equipments based on the electric energy data acquisition system. We had an experiment on meter reading between smart interaction system and electric energy data acquisition. And the test result showed that home interaction terminal could obtain the right information of smart meter, which didn't have influence on the electric energy data acquisition system. Figure 6 shows the structure of experimental demonstration system. In the electric energy data acquisition system, concentrator read 51 smart meters' electric energy on a continuous loop. Meanwhile we launched the interaction system that each of the 13 home interaction terminals read their smart meters' electric energy at certain interval. 


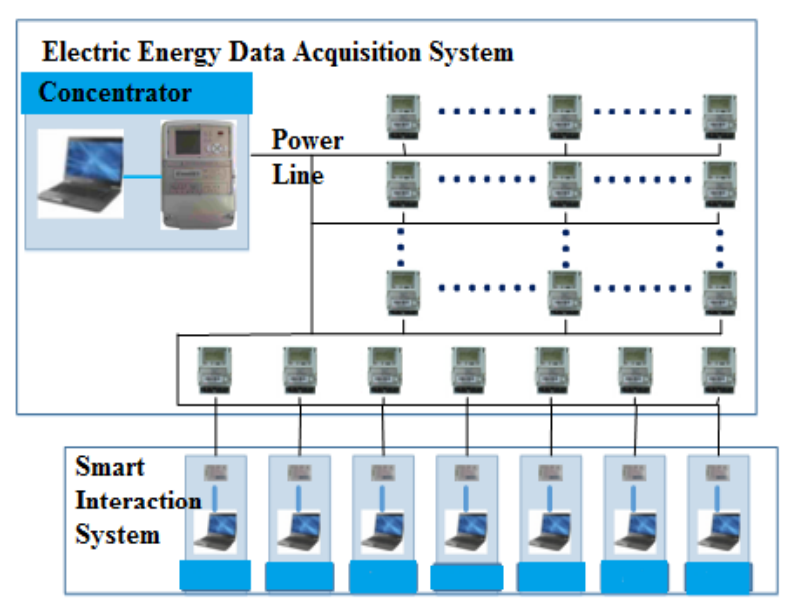

Figure 6. The structure of experimental demonstration system.

A. Success rate test of meter reading with the concentrator

The concentrator read 51 smart meters' electric energy 30 times on a continuous loop, and the interactive system stopped operating. The concentrator took 489s to read meters 1530 times totally, and the meter reading success rate was $100 \%$. The test result is shown in the table 2.

TABLE II. TEST RESULT OF METER READING WITH THE CONCENTRATOR

\begin{tabular}{|c|c|c|}
\hline No. of data items & Success rate(\%) & Time - consuming (s) \\
\hline 1530 & 100 & 554 \\
\hline
\end{tabular}

B. Success rate test of meter reading with home interaction terminal and the concentrator

The concentrator and interactive system worked simultaneously, and each of 13 home interaction terminals read meters at 3s, 5s, 10s interval. Table 3 and table 4 shows the test result of home interaction terminal and the concentrator read meters simultaneously.

TABLE III. TEST RESULT OF METER READING WITH THE CONCENTRATOR

\begin{tabular}{|c|c|c|}
\hline No. of data items & Success rate(\%) & Time - consuming (s) \\
\hline 1530 & 100 & 554 \\
\hline 1530 & 100 & 543 \\
\hline 1530 & 100 & 516 \\
\hline
\end{tabular}

TABLE IV. TEST RESULT OF METER READING WITH HOME INTERACTION TERMINAL

\begin{tabular}{|c|c|c|}
\hline Interval of meter reading $(\mathbf{s})$ & No. of data items & Success rate(\%) \\
\hline 3 & 2571 & 98.25 \\
\hline 5 & 1519 & 98.35 \\
\hline 10 & 768 & 98.70 \\
\hline
\end{tabular}

From test $\mathrm{A}$ and $\mathrm{B}$, we can obtain the following conclusions:

1)The communication success rate of home interaction terminal could reach $98.25 \%$ in the test situation.

2)The home interaction terminal did not affect the success rate of electric energy data acquisition system.

3)With home interaction terminal's operating frequency increasing, the communication success rate decreased slightly, but it did not affect the performance of smart interaction system and electric energy data acquisition system.

\section{Conclusion}

In this paper, we addressed a smart interaction system based on the power line broadband carrier technology. And the important thing is that we had the study and exploitation on the home interaction terminal, which was used for smart grid at home, which provided a smart interactive platform for power users and grid. Users could obtain the date collection of meters by smart phones, tablets, and receive the announcements from master station. On the other hand, home interaction terminal could monitor and control electric equipments at home, and help users to achieve reasonable 
electric energy. At last, we had a meter reading test between electric energy data acquisition system and home interaction terminal, the test result verified the feasibility and superiority of this project that electric energy data acquisition system could be transformed to the smart interaction system.

\section{References}

[1] Hu Jiangyi, Zhu Enguo, Du Xingang, and Du Shuwei. "The State of the Art and Developing Trend for the Researches and Applications of electric energy data acquisition system”, Automation of Electric Power Systems,vol.38, no.2, pp.131-135, 2014.

[2] Yang Shun, and Li Mingming, "Design of Remote Automatic Meter Reading System Based on ARM and WiFi Technology”, Computer Measurement \& Control, vol.21, no.11, pp.3068-3071, 2013.

[3] Qi Jiajin, Chen Xueping, and Liu Xiaosheng, “Advances of Research on Low-Voltage Power Line Carrier Communication Technology“, Power System Technology, vol.34, no.5, pp.161-172, 2010.

[4] Zhang Luhua, Wang Sitong, Yuan Ruiming, Zhou Hui, and Yin Qingduo, "Effects of Advanced Metering Infrastructure(AMI) on Relations of Power Supply and Application in Smart Grid“, Electrical Measurement \& Instrumentation, vol.47, no.537, pp. 35-38,2010.

[5] Zhang Baohui, Fu Keyuan, Zheng Tao, Liang Dong, Wang Long, and Yan Chenguang, "Conception and technology for the smart(autonomous) internet of power consumption equipments part3: key technologies of power line communications”, Power System Protection and Control, vol.41, no.8, pp.1-6, 2013.

[6] Lili Sun, Xin Lu, and Yeshen He,”Research on Power User Electrical Energy Data Acquire System Based on Micro-Power Wireless Technology”,Applide Mechanics and Materials, vol.494495, pp.1895-1901,2014. 